\title{
Sulforaphane reverses gefitinib tolerance in human lung cancer cells via modulation of sonic hedgehog signaling
}

\author{
FANPING WANG ${ }^{1,2^{*}}$, WENJUN WANG ${ }^{3 *}$, JUNPENG LI $^{4}$, \\ JINGJING ZHANG $^{1}$, XIA WANG ${ }^{1}$ and MINGYONG WANG ${ }^{1}$ \\ ${ }^{1}$ School of Laboratory Medicine, Xinxiang Medical University; ${ }^{2}$ Collaborative Innovation Center of \\ Molecular Diagnosis and Laboratory Medicine in Henan Province, Xinxiang, Henan 453003; \\ ${ }^{3}$ Cancer Research Institute, Sun Yat-sen Medical University, Guangzhou, Guangdong 510515; \\ ${ }^{4}$ Department of Clinical Laboratory, The Third Affiliated Hospital of Xinxiang \\ Medical University, Xinxiang, Henan 453003, P.R. China
}

Received November 5, 2015; Accepted August 17, 2017

DOI: $10.3892 / \mathrm{ol} .2017 .7293$

\begin{abstract}
Gefitinib is a targeted anticancer drug that was developed as an effective clinical therapy for lung cancer. Numerous patients develop gefitinib resistance in response to treatment. Sulforaphane (SFN) is present in cruciferous vegetables, and has been demonstrated to inhibit the malignant growth of various types of cancer cells. To investigate the role of SFN in gefitinib resistance, a gefitinib-tolerant PC9 (PC9GT) cell model was established by continually exposing PC9 cells to gefitinib. Cell viability was measured using a cell proliferation assay. Components of the sonic hedgehog (SHH) signaling pathway and markers of lung cancer stem cells were detected via western blotting. SFN markedly inhibited the proliferation of PC9GT and PC9 cells in a dose-dependent manner; combination SFN/gefitinib treatment also markedly decreased PC9GT cell proliferation, compared with SFN or gefitinib administered alone $(\mathrm{P}<0.05)$. Western blot analysis revealed that the expression of SHH, Smoothened (SMO), zinc finger protein GLI1 (GLI1), GLI2, CD133 and CD44 were upregulated in PC9GT cells, as compared with in PC9 cells. Furthermore, SFN markedly inhibited the expression
\end{abstract}

Correspondence to: Dr Mingyong Wang, School of Laboratory Medicine, Xinxiang Medical University, 601 Jinsui Road, Xinxiang, Henan 453003, P.R. China

E-mail: wangfanping118@126.com

*Contributed equally

Abbreviations: SFN, sulforaphane; PC9GT, gefitinib-tolerant PC9 cells; NSCLC, non-small cell lung cancer; EGFR, epidermal growth factor receptor; TKI, tyrosine kinase inhibitor; $\mathrm{SHH}$, sonic hedgehog; CSC, cancer stem cell

Key words: lung cancer, sulforaphane, gefitinib tolerance, sonic hedgehog signaling pathway of SHH, SMO and GLI1 in PC9GT and PC9 cells in a dose dependent manner, and SFN combined with gefitinib markedly inhibited the expression of SHH, SMO, GLI1, CD133 and CD44 in PC9GT cells when compared with SFN or gefitinib monotherapy. The results of the present study demonstrated that SFN inhibits the proliferation of gefitinib-tolerant lung cancer cells via modulation of the SHH signaling pathway. Therefore, combined SFN and gefitinib therapy may be an effective approach for the treatment of lung cancer.

\section{Introduction}

Lung cancer is the most prevalent and lethal type of cancer worldwide (1). Non-small cell lung cancer (NSCLC) is the most common subtype of lung cancer, accounting for $85 \%$ of cases globally in $2012(2,3)$. Gefitinib is an epidermal growth factor receptor (EGFR)-associated tyrosine kinase inhibitor (TKI) (4), and is an anticancer targeted drug that was developed as an effective clinical therapy for patients with NSCLC. However, data from clinical studies indicate that numerous patients with cancer develop gefitinib resistance, leading to poorer treatment outcomes (5), and this acquired resistance limits the further applications of the drug. Therefore, improving gefitinib efficacy and to increasing the benefits of TKI therapy conferred to patients with NSCLC is of clinical importance.

Sulforaphane (SFN) is an isothiocyanate that exists as conjugates in the genus Brassica of cruciferous vegetables; SFN has been demonstrated to inhibit the malignant growth of various cancer cell types with little or no toxicity towards normal cells $(6,7)$. SFN is a potent anticancer agent, but its underlying mechanisms and molecular targets remain unclear.

Hedgehog signaling has an essential role in the control of stem cell growth in embryonic tissues, and it is essential for the development of tissues and organs (8). The sonic hedgehog (SHH) signaling pathway controls cell proliferation and differentiation during embryonic development, and contributes to tumorigenesis when mutated or dysregulated (9). Furthermore, aberrant activation of the SHH signaling pathway serves a 
critical role in the tumorigenesis and progression of lung cancer (10-12).

Cancer stem cells (CSCs) (13) are a rare population of undifferentiated tumorigenic cells responsible for tumor initiation, maintenance and metastasis. These cells exhibit unlimited proliferation potential, self-renewal and the capacity to generate a progeny of differentiated cells that constitute the major tumor population. CSCs are more resistant to standard chemotherapy drugs, and employ various signaling pathways (14-16). Rodova et al (17) reported that SHH signaling regulates the self-renewal of pancreatic CSCs. Eramo et al (18) dissociated $\mathrm{CD}_{133^{+}}$cells from lung tumor tissue specimens, and confirmed these cells have characteristics of CSCs. Additionally, they identified that $\mathrm{CD} 133^{+}$cells are resistant to conventional chemotherapy. CD44 was also defined as a surface marker of CSCs (15). Therefore, the membrane antigens CD133 and CD44 are shared among CSCs (19-22).

Cross-talk between the SHH and epidermal growth factor receptor (EGFR) pathways involved in carcinogenesis have recently been examined; these pathways co-operate during disease initiation and progression, resulting in aggressive metastasis (23). A prior study investigated the co-targeting of the SHH and EGFR signaling pathways as a novel approach to overcoming treatment resistance and eliminating CSCs $(24,25)$.

It has reported that SFN inhibits the expression of key $\mathrm{SHH}$ factors in numerous cancer types $(7,17,26)$. However, it is unclear whether SFN can reverse gefitinib resistance in human lung cancer cells. The present study was based on the hypothesis that SFN reverses gefitinib tolerance in lung cancer cells by modulating the SHH signaling pathway. The present study aimed to explore the potential molecular mechanisms of SFN, and the feasibility of utilizing SFN to reverse gefitinib resistance in human lung cancer cells via targeting the $\mathrm{SHH}$ signaling pathway. The current study also aimed to provide experimental evidence for subsequent clinical applications, and to identify novel agents effective in the treatment of gefitinib-resistant lung cancer.

\section{Materials and methods}

Cell culture. The NSCLC PC9 cells were provided by the Cancer Institute of Southern Medical University (Guangzhou, China), and maintained in RPMI-1640 medium (Invitrogen; Thermo Fisher Scientific, Inc., Waltham, MA, USA) with $10 \%$ fetal bovine serum (HyClone; GE Healthcare Life Sciences, Logan, UT, USA) at $37^{\circ} \mathrm{C}$ in a humidified atmosphere of $5 \%$ $\mathrm{CO}_{2}$.

Establishment of a gefitinib-tolerantcell line. Gefitinib-tolerant cells were developed through chronic, repeated exposure to gefitinib. Briefly, PC9 cells were exposed to $0.002 \mu \mathrm{mol} / 1$ gefitinib for $48 \mathrm{~h}$ in RPMI-1640 medium containing $10 \%$ fetal bovine serum. Cells were then washed and cultured in drug-free medium until they reached the logarithmic growth phase. Subsequently, the cells were re-exposed to increasing concentrations $(0.002,0.020,0.050,0.100,0.250,0.500$, $1.000,2.000 \mu \mathrm{mol} / \mathrm{l})$ of gefitinib. Resistant cells can survive in $2 \mu \mathrm{mol} / 1$ gefitinib; gefitinib-tolerant cells were obtained 48 days after initial exposure. The established resistant cell line was maintained in RPMI-1640 medium containing $1 \mu \mathrm{mol} / 1$ gefitinib. For all studies, resistant cells were cultured in drug-free medium for 1 week to eliminate gefitinib. Gefitinib-tolerant cells are referred as PC9GT cells.

Cell proliferation assay. The PC9 and PC9GT cells, used in the logarithmic phase of growth, were seeded into 96-well plates (3,000 cells/well). When the cells had adhered, various concentrations of gefitinib were added to the wells; the final concentrations were $0.002,0.020,0.050,0.100,0.250,0.500$, 1.000 and $2.000 \mu \mathrm{mol} / 1$. Following incubation for $48 \mathrm{~h}, 10 \mu \mathrm{l}$ MTT reagent was added into each well of the plates. After an additional incubation period of $4 \mathrm{~h}, 150 \mu \mathrm{l}$ DMSO reagent was added, and then the optical density (OD) at $490 \mathrm{~nm}$ was determined using a micro plate reader (Beckman Coulter, Inc., Brea, CA, USA). Each drug concentration was evaluated in triplicate wells. The number of cells was counted using Countstar (version IC-1000; Rui Yu Biological Technology Co., Ltd., Shanghai, China). The density of cells was observed with a inverted microscope system (magnification, $\mathrm{x} 40$; Eclipse Ti-s, Nikon, Tokyo, Japan).

Western blot analysis. Total protein was extracted from 2-5x $10^{6}$ PC9 and PC9GT cells with an ice-cold SDS protein lysis buffer at $100^{\circ} \mathrm{C}$ for $10 \mathrm{~min}$. Protein concentration was measured using the Micro BCA Protein Assay Reagent kit (Sangon Biotech Co., Ltd., Shanghai, China). Proteins were separated using a $12.5 \%$ gel and SDS-PAGE and transferred onto polyvinylidene difluoride membranes (EMD Millipore, Billerica, MA, USA). The membranes were blocked with 5\% non-fat dry milk in TBS buffer for $1 \mathrm{~h}$ at room temperature and subsequently incubated with primary antibodies against CD133, CD44, SHH, SMO, GLI1, GLI2 and GAPDH (1:1,000; Cell Signaling Technology, Inc., Danvers, MA, USA) overnight at $4^{\circ} \mathrm{C}$, washed three times with TBST ( $0.05 \%$ Tween 20$)$, and then incubated with the secondary antibodies conjugated with horseradish peroxidase at 1:3,000 dilutions in TBS for $2 \mathrm{~h}$ at room temperature. Membranes were then washed again in TBS-Tween 20 three times at room temperature. Protein bands were visualized on an X-ray film using chemiluminescent horseradish peroxidase substrate (SurModics, Inc., Eden Prairie, MN, USA). A Tanon v.3500 Gel Imaging System (Tanon Co., Shanghai, China) was used for the semi-quantitative analysis of proteins.

Statistical analysis. Statistical analysis was performed using SPSS version 16.0 software (SPSS, Inc., Chicago, IL, USA). The data are expressed as the means \pm standard deviation. The Student's t-test was used for comparisons between two groups. Multigroup comparisons of the means were performed using one-way analysis of variance and post hoc analysis with a Student-Newman-Keuls test. $\mathrm{P}<0.05$ was considered to indicate a statistically significant difference.

\section{Results}

PC9GT cell lines were established. To generate a gefitinib-tolerant subline of PC9 cells, the cells were cultured with increasing concentrations of gefitinib over a period of 48 days. To confirm that the new cell line was established successfully, PC9GT and PC9 cells were divided into two 
A
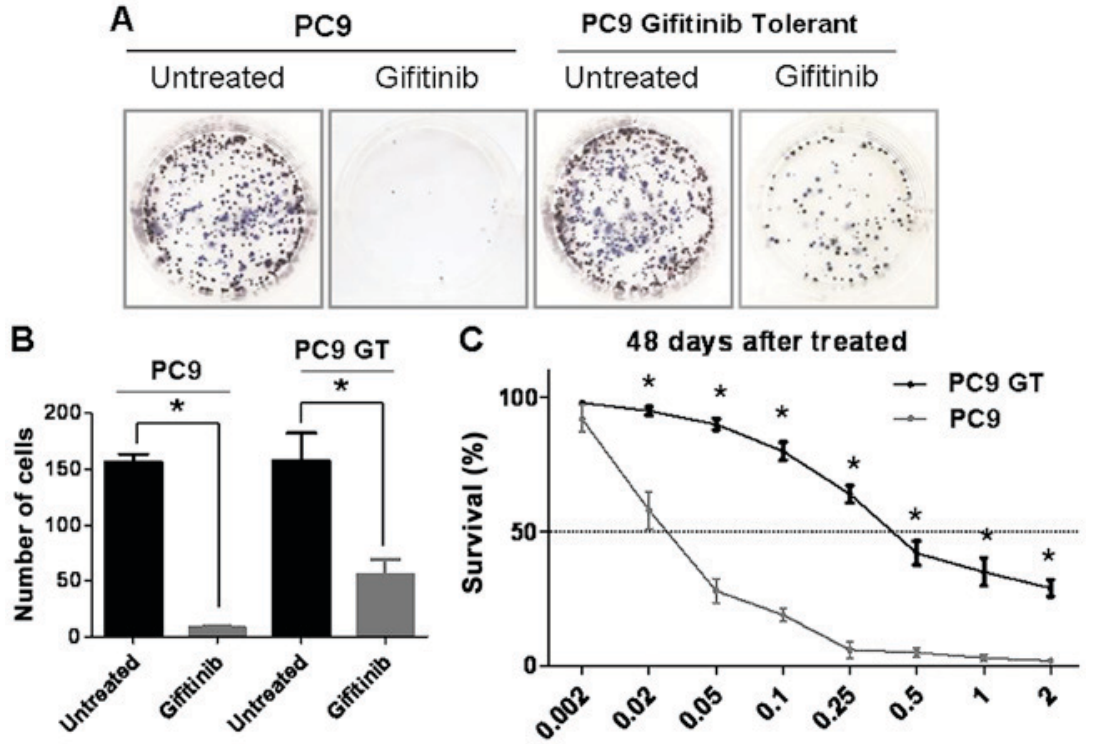

Figure 1. Sensitivity to gefitinib was determined using a cell proliferation assay. (A) PC9GT and PC9 cells were separated into two groups. One group was treated with gefitinib and the other remained untreated. (B) The cell counting assay demonstrated that PC9GT cells continued to proliferate, unlike the PC9 cells ( $\left.{ }^{*} \mathrm{P}<0.05\right)$. (C) The MTT assay showed that the PC9GT cells were able to continue proliferating when exposed to $2 \mu \mathrm{mol} / 1$ gefitinib, contrary to PC9 cells ( $\mathrm{P}<0.05$, compared with PC9 cells). SFN, sulforaphane; PC9GT, gefitinib-tolerant PC9 cells.

groups. One group was treated with gefitinib and the other remained untreated (Fig. 1A). The cell count demonstrated that PC9GT cells continued to proliferate, contrary to the PC9 cells $(\mathrm{P}<0.05$; Fig. 1B). The PC9GT cell line could be serially passed in gefitinib-free media for $>15$ generations without a change in sensitivity to gefitinib. The MTT assay showed that the PC9GT cells could proliferate in response to exposure to $2 \mu \mathrm{mol} / 1$ gefitinib, compared with PC9 cells $(\mathrm{P}<0.05$; Fig. 1C).

Activation of the SHH signaling pathway affects gefitinib sensitivity. Aberrant activation of the SHH signaling pathway serves a critical role in the tumorigenesis and progression of lung cancer. To determine whether the SHH signaling pathway is associated with gefitinib resistance, the key proteins of the SHH signaling pathway in PC9 and PC9GT cells were detected via western-blot analysis. Notably, robust expression of SHH, SMO, GLI1 and GLI2 was observed in PC9GT cells, as compared with in the PC9 cells (Fig. 2A).

The existence of CSCs affects gefitinib sensitivity. The presence of CSCs in lung cancer may render standard chemotherapy ineffective and aid the cancer cells in resisting anti-cancer drugs (27). Previous reports suggest that a CD133- and CD44-positive subpopulation of multipotent cells, with extensive proliferative and self-renewal characteristics, have the biological features of CSCs $(19,20)$. Mizugaki et al (21) reported that the expression of CD133 is a potential prognostic marker for NSCLC. CD133 and CD44 have been established as representative markers for CSC in diverse types of cancer (28). To determine whether the CSCs were associated with gefitinib resistance, the protein expression of CD133 and CD44 were examined using western blotting. The expression of CD133 and CD44 was upregulated in PC9GT cells, compared with in PC cells (Fig. 2B). Upregulated CD133and CD44 were also revealed to be

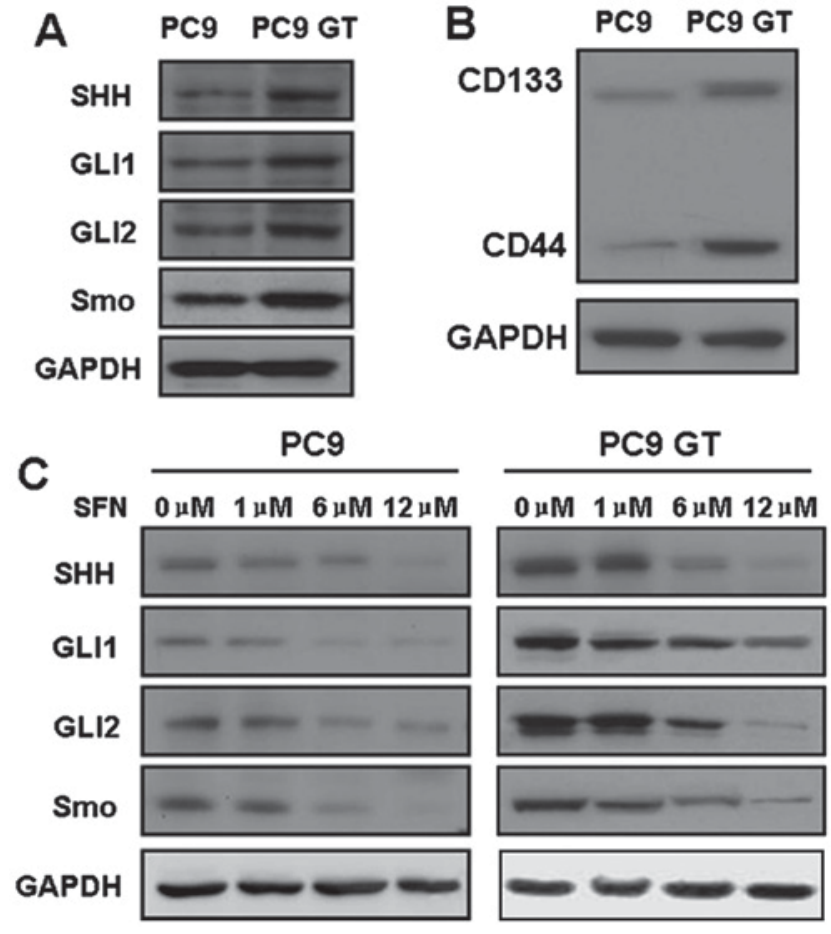

Figure 2. The expression of key SHH and the marker of LSCs in lung cancer cells was analyzed via western blotting. (A) The expression of SHH, SMO, GLI1 and GLI2 was high in PC9GT cells, as compared with in PC9 cells. (B) The expression of CD133 and CD44 was upregulated in PC9GT cells, compared with in PC9 cells. (C) SFN markedly inhibited the expression of SHH, SMO and GLI1 in PC9GT and PC9 cells, in a dose-dependent manner. SFN, sulforaphane; PC9GT, gefitinib-tolerant PC9 cells; EGFR, epidermal growth factor receptor; SHH, sonic hedgehog; SMO, smoothened; GLI1/2, zinc finger protein GLI1/2.

associated with significantly poorer prognosis for NSCLC. Upregulated expression of CD133 and CD44 could therefore be associated with gefitinib resistance. 


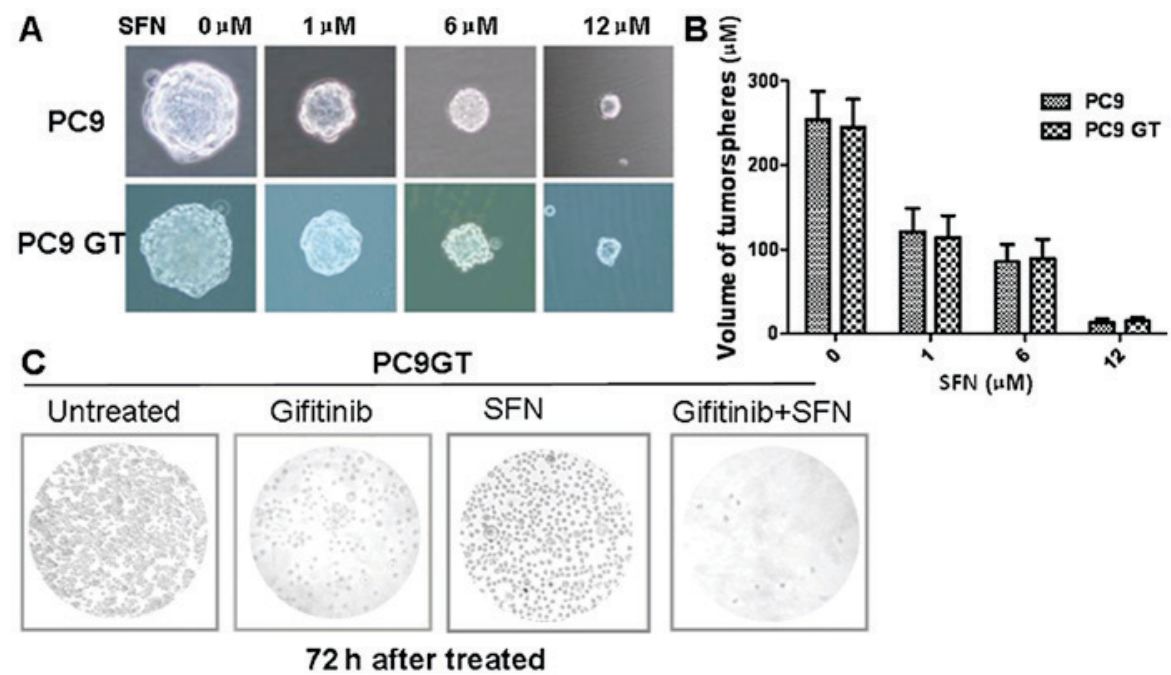

Figure 3. PC9 and PC9GT cell proliferation was affected by SFN treatment. (A) SFN markedly inhibited the proliferation of PC9GT and PC9 in a dose-dependent manner. (B) The tumorsphere volumes were not significantly different between the PC9GT and PC9 cells. (C) PC9GT cells were treated with gefitinib, SFN and combined therapy with SFN and gefitinib. Combined treatment markedly decreased PC9GT cell density, as observed by microscopy. SFN, sulforaphane; PC9GT, gefitinib-tolerant PC9 cells.

A

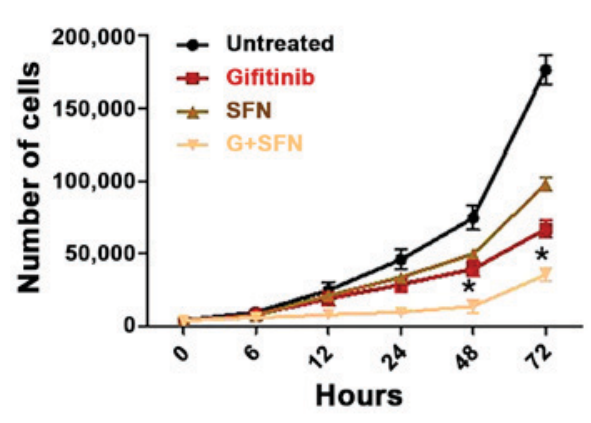

B

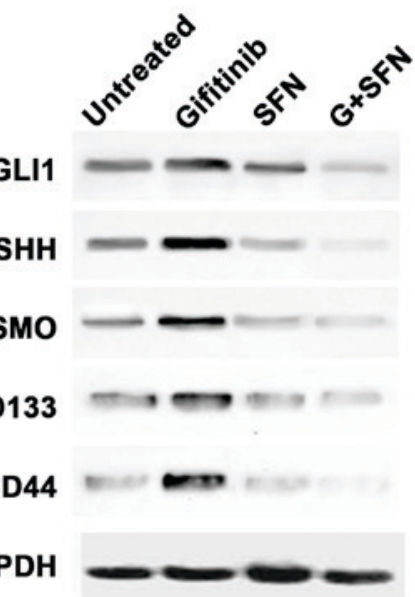

Figure 4. The effect of combined SFN and gefitinib therapy on PC9GT cells was determined using a cell proliferation assay and western blotting. (A) Combined treatment markedly decreased PC9GT cell proliferation, when compared with SFN or gefitinib monotherapy, at 48 and 72 h ( $\mathrm{P}<0.05$, compared with SFN, gefitinib and the control). (B) SFN combined with gefitinib markedly inhibited the expression of SHH, SMO, GLI1, CD133 and CD44 in PC9GT cells, as compared with SFN or gefitinib administered alone. SFN, sulforaphane; PC9GT, gefitinib-tolerant PC9 cells; EGFR, epidermal growth factor receptor; SHH, sonic hedgehog; SMO, Smoothened; GLI1, zinc finger protein GLI1.

Sulforaphane inhibits PC9 and PC9GT cell proliferation and enhances the chemotherapeutic effect of gefitinib. A sphere formation assay was performed to investigate whether SFN sensitizes gefitinib-tolerant lung cancer cells. The PC9GT cells and PC9 cells were treated with $0,1,6$ and $12 \mu \mathrm{mol} / 1$ SFN. After 1 week, the total number of spheres and the tumorsphere volumes were measured; the tumorsphere volumes were observed to gradually decrease with the increasing dose of SFN (Fig. 3A). SFN markedly inhibited the proliferation of PC9GT and PC9 cells in a dose-dependent manner, although there was no significant difference between the PC9GT and PC9 cells (Fig. 3B), indicating that SFN can effectively inhibit lung cancer cell proliferation, and is unaffected by gefitinib-resistance.

The effects of combined therapy with SFN and gefitinib were then assessed in PC9GT cells. The PC9GT cells were treated with SFN, gefitinib or combined SFN/gefitinib. The combined treatment was observed to markedly decrease PC9GT cell proliferation, when compared with SFN or gefitinib administered alone (Fig. 3C). The combined treatment significantly decreased PC9GT cell proliferation, when compared with SFN or gefitinib alone, after 48 and $72 \mathrm{~h}$ ( $\mathrm{P}<0.05$; Fig. 4A).

Sulforaphane reverses gefitinib resistance via modulation of the SHH signaling pathway. To explore the mechanism underlying the anti-proliferative effect of SFN on PC9GT cells, the SHH signaling pathway proteins CD133 and CD44 were examined via western blotting in SFN-treated PC9 and PC9GT cells. Notably, SFN markedly inhibited the expression of SHH, SMO and GLI1 in PC9GT and PC9 cells in a dose-dependent manner. Furthermore, the expression of these 
proteins did not significantly differ between the PC9GT and PC9 cells (Fig. 2C). The effects of combined SFN/gefitinib therapy PC9GT cells were then assessed. The results indicate that SFN combined with gefitinib markedly inhibits the expression of SHH, SMO, GLI1, CD133 and CD44 in PC9GT cells, when compared with SFN or gefitinib administered alone (Fig. 4B). The results suggested that SFN may reverse gefitinib resistance by inhibiting the $\mathrm{SHH}$ pathway. In the present study, it was also identified that SFN can inhibit the expression of CD133 and CD44 in PC9GT cells.

\section{Discussion}

Gefitinib is effective for patients with NSCLC, but numerous patients develop gefitinib resistance, which leads to poor treatment outcomes. Acquired resistance to gefitinib is a serious clinical problem; therefore, the clinical use of gefitinib has been limited.

SFN exists as conjugates in cruciferous vegetables, and has been demonstrated to inhibit the malignant growth of cancer cells with little or no toxicity towards normal cells. The present study evidenced that SFN may inhibit the proliferation of PC9 cells, but also inhibit the proliferation of PC9 GT cells. The present study could be important in searching for a novel potential agent against gefitinib-resistant lung cancer.

Further studies are required to clarify the mechanism underlying SFN-mediated inhibition of gefitinib resistance. The results indicated that components of the SHH signaling pathway are highly expressed in PC9GT cells. To identify the function of SFN in the SHH signaling pathway, the expression of SHH, GLI1, GLI2 and SMO was measured in PC9GT cells following treatment with SFN, gefitinib and SFN and gefitinib. The present study suggested that SFN may inhibit the expression of certain SHH signaling pathway-associated proteins, including SHH, GLI1, GLI2 and SMO, in PC9GT cells. Gefitinib is an inhibitor of the EGFR-associated TK, and exerts antitumor effects via the blockade of EGFR-associated TK activity (29). It is evident the SHH signaling pathway co-operates with EGFR during cancer initiation, and the progression to aggressive, invasive and metastatic disease stages $(23,24)$. Concordantly, combined therapy with the $\mathrm{SHH}$ signaling pathway antagonist and the EGFR inhibitor would be more efficient in inhibiting invasiveness and inducing cancer cell apoptosis compared with either drug alone. Therefore, combining SFN and gefitinib meets the aforementioned requirements; the effectiveness of SFN in inhibiting PC9GT cell growth may be associated with activation of the $\mathrm{SHH}$ signaling pathways.

Recent studies have suggested that $\mathrm{SHH}$ serves a critical role in maintaining the CSC pool (30). CD44 and CD133 are defined as CSC surface markers $(18,31)$. Lung cancer may resist conventional chemotherapy due to the presence of the rare CSC (31). CSCs are not only resistant to standard chemotherapy drugs, but are also associated with multiple signaling pathways, including $\mathrm{HH}$, Notch and Wnt signaling pathways $(15,32,33)$. A previous study demonstrated that increased CD133 expression was associated with chemoresistance and poorer clinical outcomes in lung cancer (34). The present study revealed that the expression of CD44 and CD133 was increased in PC9GT cells and examined whether the presence of lung CSCs could help explain gefitinib resistance in lung cancer.

Recent studies underlining the therapeutic interest of co-targeting the $\mathrm{SHH}$ and EGFR signaling pathways, and eradicating CSCs/progenitor cells, may improve current clinical therapies against aggressive and metastatic cancer cases. In the current study, it was identified that SFN is effective against gefitinib-resistant lung cancer cells and lung CSCs. SFN may inhibit the proliferation of gefitinib-resistant lung cancer cells via modulating the SHH signaling pathway. Furthermore, combined treatment markedly decreases PC9GT cell proliferation when compared with SFN or gefitinib monotherapy. A reason SFN inhibits the proliferation of gefitinib-tolerant lung cancer cells may be due to its modulation of SHH signaling pathway activation and the inhibition of lung CSC self-renewal.

The results of the present study will facilitate the identification of novel drug targets and the development of new therapeutic strategies to block this tumorigenic cascade, thus improving currently available cancer treatments.

To conclude, SFN was identified to be a potent anticancer agent: In addition to inhibiting lung cancer cell proliferation, it also kills gefitinib-tolerant cells. The data demonstrated that SFN reverses gefitinib tolerance in human lung cancer cells by modulating the SHH signaling pathway. Furthermore, the combination of SFN and gefitinib could be an effective treatment approach for lung cancer.

\section{Acknowledgements}

The present study was supported by The Program for New Century Excellent Talents in University of China (grant no. NCET-13-0990), The Project of Science and Technology Innovation Talents in Universities of Henan Province (grant no. 2012HASTIT024) and The Project of Science and Technology of Education Department of Henan Province (grant nos. 15A320009 and 13B320220). Special thanks are given to Dr Prajaya Shikhar Shrestha (National Academy of Medical Science, Bir Hospital, Kathmandu, Nepal) for their dedicated revision of the manuscript.

\section{References}

1. Siegel R, Ma J, Zou Z and Jemal A: Cancer statistics, 2014. CA Cancer J Clin 64: 9-29, 2014

2. Meoni G, Cecere FL, Lucherini E and Di Costanzo F: Medical treatment of advanced non-small cell lung cancer in elderly patients: A review of the role of chemotherapy and targeted agents. J Geriatr Oncol 4: 282-290, 2013.

3. Puglisi M, Thavasu P, Stewart A, de Bono JS, O'Brien ME, Popat S, Bhosle $\mathrm{J}$ and Banerji U: AKT inhibition synergistically enhances growth-inhibitory effects of gefitinib and increases apoptosis in non-small cell lung cancer cell lines. Lung Cancer 85: 141-146, 2014.

4. Jung SK, Lee MH, Lim DY, Lee SY, Jeong CH, Kim JE, Lim TG, Chen H, Bode AM, Lee HJ, et al: Butein, a novel dual inhibitor of MET and EGFR, overcomes gefitinib-resistant lung cancer growth. Mol Carcinog 54: 322-331, 2015.

5. Ge X, Chen Q, Wu YP, Zhang Y, Xia H, Yuan D, Chen Q, Leng W, Chen L, Tang Q, et al: Induced IGF-1R activation contributes to gefitinib resistance following combined treatment with paclitaxel, cisplatin and gefitinib in A549 lung cancer cells. Oncol Rep 32: 1401-1408, 2014.

6. Clarke JD, Dashwood RH and Ho E: Multi-targeted prevention of cancer by sulforaphane. Cancer Lett 269: 291-304, 2008. 
7. Priya DK, Gayathri R, Gunassekaran G, Murugan S and Sakthisekaran D: Chemopreventive role of sulforaphane by upholding the GSH redox cycle in pre- and post-initiation phases of experimental lung carcinogenesis. Asian Pac J Cancer Prev 12: 103-110, 2011.

8. Varjosalo M and Taipale J: Hedgehog: Functions and mechanisms. Genes Dev 22: 2454-2472, 2008.

9. Shi S, Deng YZ, Zhao JS, Ji XD, Shi J, Feng YX, Li G, Li JJ, Zhu D, Koeffler HP, et al: RACK1 promotes non-small-cell lung cancer tumorigenicity through activating sonic hedgehog signaling pathway. J Biol Chem 287: 7845-7858, 2012.

10. Raz G, Allen KE, Kingsley C, Cherni I, Arora S, Watanabe A, Lorenzo CD, Edwards VD, Sridhar S, Hostetter G and Weiss GJ: Hedgehog signaling pathway molecules and ALDH1A1 expression in early-stage non-small cell lung cancer. Lung Cancer 76: 191-196, 2011.

11. Vestergaard J, Pedersen MW, Pedersen N, Ensinger C, Tümer Z, Tommerup N, Poulsen HS and Larsen LA: Hedgehog signaling in small-cell lung cancer: Frequent in vivo but a rare event in vitro. Lung Cancer 52: 281-290, 2006.

12. Maitah MY, Ali S, Ahmad A, Gadgeel S and Sarkar FH Up-regulation of sonic hedgehog contributes to TGF-betal-induced epithelial to mesenchymal transition in NSCLC cells. PLoS One 6 : e16068, 2011.

13. Allegra A, Alonci A, Penna G, Innao V, Gerace D, Rotondo F and Musolino C: The cancer stem cell hypothesis: A guide to potential molecular targets. Cancer Invest 32: 470-495, 2014.

14. Tanaka H, Nakamura M, Kameda C, Kubo M, Sato N, Kuroki S, Tanaka $\mathrm{M}$ and Katano M: The Hedgehog signaling pathway plays an essential role in maintaining the CD44+CD24-/low subpopulation and the side population of breast cancer cells. Anticancer Res 29: 2147-2157, 2009.

15. Liu M, Inoue K, Leng T, Guo S and Xiong ZG: TRPM7 channels regulate glioma stem cell through STAT3 and Notch signaling pathways. Cell Signal 26: 2773-2781, 2014.

16. Arasada RR, Amann JM, Rahman MA, Huppert SS and Carbone DP: EGFR blockade enriches for lung cancer stem-like cells through Notch3-dependent signaling. Cancer Res 74 $5572-5584,2014$

17. Rodova M, Fu J, Watkins DN, Srivastava RK and Shankar S Sonic hedgehog signaling inhibition provides opportunities for targeted therapy by sulforaphane in regulating pancreatic cancer stem cell self-renewal. PLoS One 7: e46083, 2012.

18. Eramo A, Lotti F, Sette G, Pilozzi E, Biffoni M, Di Virgilio A, Conticello C, Ruco L, Peschle C and De Maria R: Identification and expansion of the tumorigenic lung cancer stem cell population. Cell Death Differ 15: 504-514, 2008

19. Okudela K, Woo T, Mitsui H, Tajiri M, Masuda M and Ohashi K: Expression of the potential cancer stem cell markers, CD133, CD 44, ALDH1, and $\beta$-catenin, in primary lung adenocarcinoma-their prognostic significance. Pathol Int 62: 792-801, 2012

20. Kitamura H, Okudela K, Yazawa T, Sato H and Shimoyamada H: Cancer stem cell: Implications in cancer biology and therapy with special reference to lung cancer. Lung Cancer 66 : 275-281, 2009

21. Mizugaki H, Sakakibara-Konishi J, Kikuchi J, Moriya J, Hatanaka KC, Kikuchi E, Kinoshita I, Oizumi S, Dosaka-Akita H, Matsuno Y and Nishimura M: CD133 expression: A potential prognostic marker for non-small cell lung cancers. Int J Clin Oncol 19: 254-259, 2014
22. Shimada M, Sugimoto K, Iwahashi S, Utsunomiya T, Morine Y, Imura S and Ikemoto T: CD133 expression is a potential prognostic indicator in intrahepatic cholangiocarcinoma. J Gastroenterol 45: 896-902, 2010.

23. Heo JS, Lee MY and Han HJ: Sonic hedgehog stimulates mouse embryonic stem cell proliferation by cooperation of $\mathrm{Ca} 2+/$ protein kinase $\mathrm{C}$ and epidermal growth factor receptor as well as Gli1 activation. Stem Cells 25: 3069-3080, 2007.

24. Kasper M, Schnidar H, Neill GW, Hanneder M, Klingler S, Blaas L, Schmid C, Hauser-Kronberger C, Regl G, Philpott MP and Aberger F: Selective modulation of Hedgehog/GLI target gene expression by epidermal growth factor signaling in human keratinocytes. Mol Cell Biol 26: 6283-6298, 2006.

25. Mimeault M and Batra SK: Frequent deregulations in the hedgehog signaling network and cross-talks with the epidermal growth factor receptor pathway involved in cancer progression and targeted therapies. Pharmacol Rev 62: 497-524, 2010.

26. Kalpana Deepa Priya D, Gayathri R and Sakthisekaran D: Role of sulforaphane in the anti-initiating mechanism of lung carcinogenesis in vivo by modulating the metabolic activation and detoxification of benzo(a)pyrene. Biomed Pharmacother 65: 9-16, 2011.

27. MacDonagh L, Gray SG, Breen E, Cuffe S, Finn SP, O'Byrne KJ and Barr MP: Lung cancer stem cells: The root of resistance. Cancer Lett 372: 147-156, 2016.

28. Kinugasa Y, Matsui T and Takakura N: CD44 expressed on cancer-associated fibroblasts is a functional molecule supporting the stemness and drug resistance of malignant cancer cells in the tumor microenvironment. Stem Cells 32: 145-156, 2014.

29. Lee CG, McCarthy S, Gruidl M, Timme C and Yeatman TJ: MicroRNA-147 induces a mesenchymal-to-epithelial transition (MET) and reverses EGFR inhibitor resistance. PLoS One 9: e84597, 2014

30. Yao J, An Y, Wie JS, Ji ZL, Lu ZP, Wu JL, Jiang KR, Chen P, Xu ZK and Miao Y: Cyclopamine reverts acquired chemoresistance and down-regulates cancer stem cell markers in pancreatic cancer cell lines. Swiss Med Wkly 141: w13208, 2011.

31. Dean M, Fojo T and Bates S: Tumour stem cells and drug resistance. Nat Rev Cancer 5: 275-284, 2005.

32. Sarvi S, Mackinnon AC, Avlonitis N, Bradley M, Rintoul RC, Rassl DM, Wang W, Forbes SJ, Gregory CD and Sethi T: CD133+ cancer stem-like cells in small cell lung cancer are highly tumorigenic and chemoresistant but sensitive to a novel neuropeptide antagonist. Cancer Res 74: 1554-1565, 2014.

33. Yang J, Chen J, He J, Li J, Shi J, Cho WC and Liu X: Wnt signaling as potential therapeutic target in lung cancer. Expert Opin Ther Targets 20: 999-1015, 2016.

34. Kubo T, Takigawa N, Osawa M, Harada D, Ninomiya T, Ochi N, Ichihara E, Yamane H, Tanimoto M and Kiura K: Subpopulation of small-cell lung cancer cells expressing CD133 and CD87 show resistance to chemotherapy. Cancer Sci 104: 78-84, 2013.

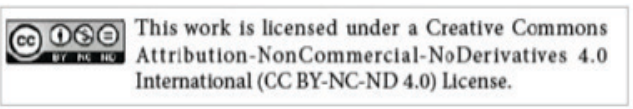

